\title{
About compiled catalogue of spectroscopic determined chemical abundances for stars with accurate parallaxes. Magnesium
}

\author{
Tatyana V. Borkova ${ }^{1}$ \\ Vladimir A. Marsakov ${ }^{1}$ \\ ${ }^{1}$ Spas Reseach Department, Rostov State University of Rossia, Sorge st., 5, 344090, Russia \\ email: borkova@ip.rsu.ru, marsakov@ip.rsu.ru
}

\begin{abstract}
We present a compiled catalogue of effective temperatures, surface gravities iron and magnesium abundances, along with distances, velocity components, and orbital elements for stars in the solar vicinity. Abundances were found from 1412 determinations in 31 publications for 876 dwarfs and subgiants by means of a three-step iteration averaging procedure, with weights assigned to each source of data as well as to each individual determination. The assumed coverage completeness for data sources containing more than 5 stars, as of late 2003, exceeds $90 \%$. For the vast majority of stars in the catalogue, the spatial-velocity components were derived from modern high-precision astrometric observations, and their galactic-orbit elements were computed using a three-component model of the Galaxy, consisting of a disk, a bulge, and a massive extended halo.
\end{abstract}

Keywords. Stars: abundances.

\section{Introduction}

Published abundances of the same chemical element for a given star often differ quite noticeably even in the case of similarly high quality of the spectra reduced by different authors. In our study, we collected all accessible lists ( $\geqslant 5$ stars) of determinations of the relative magnesium abundance, $[\mathrm{Mg} / \mathrm{Fe}]$, for field stars from high-resolution spectra with high signal-to-noise ratios published after 1989. We estimate the completeness of our sample for the abundances published for solar-vicinity stars up through December 2003 to be better than $90 \%$. The raw material for this study were 36 publications containing 1809 magnesium-abundance determinations for 1027 stars. Of these stars, we retained only 876 dwarf and subdwarf stars that lie below the solid line in the Hertzsprung-Russell diagram (Fig. 1 a). This line was plotted "by eye", parallel to the zero-age main sequence, to exclude stars at more advanced evolutionary stages. This automatically rejected five publications where only giants had been studied.

We simply averaged the stellar $\left(T_{\text {eff }}\right),(\log g)$ and $[\mathrm{Fe} / \mathrm{H}]$ values for each star from the cited papers. For these parameters, we estimated uncertainties of the averages based on the scatter of the individual determinations about the computed average for each star, i.e. from the agreement of the determinations obtained by various authors. They were found equal to $\epsilon\left(T_{\text {eff }}\right)= \pm 56 \mathrm{~K}$ and $\pm 82 \mathrm{~K}$, to $\epsilon(\log g)= \pm 0.12, \pm 0.24$, and to $\epsilon([\mathrm{Fe} / H])= \pm 0.07$ dex and \pm 0.13 dex, respectively for stars with $[\mathrm{Fe} / \mathrm{H}]$ metallicities above and below -1.0 . It was found necessary to introduce the two metallicity groups because determination uncertainties of all the parameters are considerably larger for metal-deficient stars. To derive reliable abundances, we applied a three-step iteration technique for putting data together. 

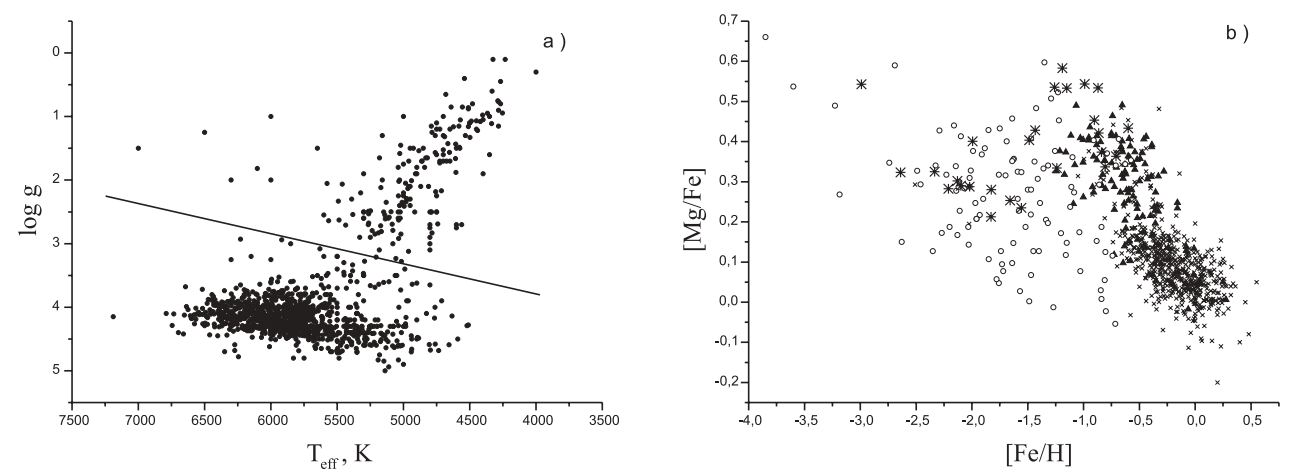

Figure 1. The $T_{\text {eff }}-\log g$ diagram for stars with magnesium abundances found in the literature (a) and the distribution of the stars in the catalog over their relative magnesium abundances(b) for the catalog's stars, divided by orbital parameters into four subsystems: dots for thin-disk stars, triangles for thick-disk stars, asterisks for proto-disk-halo stars, and open circles for accreted-halo stars. Only stars that lie above the sloping line in the diagram (a) enter our catalog

At first, we computed individual preliminary mean values, $\left\langle[\mathrm{Mg} / F e]_{i}\right\rangle$, and determined the scatter of the deviations as well as the systematic bias of $\delta[\mathrm{Mg} / F e]_{i}$ with respect of the mean. Then, we corrected all the individual $[\mathrm{Mg} / \mathrm{Fe}]_{i}$ values for these biases. Such corrections virtually did not influence the resulting magnesium abundances for stars present in several lists. However, if the star's magnesium abundance was determined in a single study only, the bias is completely present in the final magnesium abundance.

The second step was to determine the weights of the data sources in the lists inversely proportional to the corresponding deviation dispersion in each of the metallicity ranges. Then we computed for each star a new weighted mean magnesium abundance, with the revealed biases and the weights assigned to the lists taken into account.

The third step was also a weight-assigning procedure, this time to individual determinations. As a result, this procedure assigns the lowest weights to the least-reliable determinations and permits us to get final values close to those given in the majority of the sources, and with no single determination rejected. An idea on the internal accuracy of our final $[\mathrm{Mg} / \mathrm{Fe}]$ values can be obtained from the dispersion of the distribution of all the deviations equal respectively to $\epsilon([\mathrm{Mg} / \mathrm{Fe}])= \pm 0.05$ and \pm 0.07 dex for metal-rich and metal-poor stars.

In order to compute distances to the stars, we used trigonometric parallaxes with uncertainties below $25 \%$ or, in their absence, photometric distances from uvby $\beta$ photometry. Radial velocities were taken from different publications. For mean distance uncertainties of $15 \%$ and mean distances from the Sun of $\approx 60 \mathrm{pc}$ for our sample's stars, the mean uncertainty in the spatial velocity components is $\approx \pm 2 \mathrm{~km} \mathrm{~s}^{-1}$.

To compute the galactic orbital elements, we modelled 30 orbits of each star around the galactic center using the Galaxy's multi-component model, consisting of a disk, a bulge, and an extended massive halo.

Full description of the catalogue is published in the paper Borkova \& Marsakov, 2005. The catalogue itself is published only electronically, at the address http://cdsweb.ustrasbg.fr/cats/J.AZh.htx.

\section{References}

Borkova, T.V. \& Marsakov, V.A., 2005, Astron. Report, V.49,405 